\title{
Finite element analysis of circumferential crack behavior in cement-femoral prosthesis interface
}

\author{
A.A. Oshkour, M.M. Davoodi, N.A. Abu Osman, Y.H. Yau, F. Tarlochan, W.A.B. Wan Abas \\ Introduction
}

Total hip replacement $t$ (THR) has become the second most per- formed surgical procedure every year [1]. In THR, the femoral prosthesis can be fixed by either cement or cement less methods in the patient's bones. In both procedures, the stability of the prosthesis in the hosted bone plays a significant role in the long-term durability of the THR [1,2]. In the cemented femoral prosthesis, the structural strength of the THR is provided by the cement, which must withstand the mechanical stresses that may potentially result in the generation and propagation of cracks and in the eventual failure of the entire THR structure [3-5]. Therefore, analyzing the crack behavior in the cement layer is vital to the prediction $\mathrm{n}$ of fem- oral prosthesis s stability and to the prevention of cement mantle failure in the hosted bone.

Jasty et al. [5] determined that most of the cracks in the cement are initiated at the corners, or where the cement mantle is thin or incomplete. Verdonschot and Huiskes [6] reported that damage accumulation is affected by the prosthesi s-cement deboning, which significantly accelerates the failure process. Damage accumulation causes longitudinal I and radial cracks along the bounded stem and circumfe rential cracks along an unbound ed one, which is more consistent with actual conditions. Achour et al. [7] and Flitti et al. [4] presented that the mixed-mode crack propagation and the single-m ode crack opening growth occur at the distal and proximal zones of the cement layer, respectively. The majority of previous studies were performed using a two-dimensional (2D)crack anal- ysis on THR by the standard finite element method (FEM).How- ever, a FEM is a powerful tool in a multi scale analysis [8]; using the standard FEM in crack analysis has some limitations, such as mesh and singularity generation. The extended finite element method (XFEM) as apowerful tool for conducting crack analysis with minimal re-meshing was introduced by Belytschko and Black $[9,10]$. This method has shown good accuracy in measuring crack growth paths and fracture parameters [11]. Nowadays, the XFEM is widely employed in deriving stress intensity factors (SIFs). This method has been employed to compute for the SIFs in functionally graded materials [12], interface cracks [13], orthotropic biomaterial [14], bending plates [15], and sharp V-notches [16]. In the XFEM, the standard finite element shape functions are enriched locally with asymptotic c and Heaviside functions to present singular- ity at the crack tip and discontinuity $[17,18]$. The displacement vector function $u$ can be approximated with the partition of unity enrichment nt as.

Please refer to the full text

where $\mathrm{N}_{\mathrm{I}}(\mathrm{x})$ and $\mathrm{uI}$ are the standard finite element shape functions and the nodal displacement vector associateed with the continuous $\mathrm{s}$ part of the finite element $\mathrm{t}$ solution, respectively $\mathrm{y}$. ar is the addition al nodal degrees of freedom vector. $\mathrm{H}(\mathrm{x})$ is the discontinuous us jump function across the crack surfaces, which is given by

Full text is available at :

http://ac.els-cdn.com/S0261306913000605/1-s2.0-S0261306913000605-main.pdf? tid=7644b934-a018-11e3a5c2-00000aab0f6c\&acdnat $=139355144784028 c 8 a 652 \mathrm{fdbb0dbaf2c6806b4ac61}$

http://www.sciencedirect.com/science/article/pii/S0261306913000605 
